\title{
The Periods of X Cyg, T Mon, Y Oph, S Vul and SV Vul
}

\author{
A. M. Heiser \\ Dyer Observatory, Vanderbilt University, Nashville, Tennessee
}

\begin{abstract}
Photoelectric $B V$ observations have been obtained with the 16-inch Vanderbilt APT over a number of recent years for five Cepheids. These data are being examined for periods, period changes, and for possible variations in the seasonal light curves.
\end{abstract}

The Vanderbilt University APT has been used since 1989 to collect differential $B V$ data for a number of long-period Northern Hemisphere Cepheid variables. The primary purpose of this study is to obtain accurate light curves in order to compare them with other, previously acquired, photoelectric observations.

The analysis of the comparison and check star data for four of the programme stars (T Mon, Y Oph, S Vul and SV Vul) has shown that the average errors of the differential data during the total time period of our APT observations varied from 0.005 to $0.008 \mathrm{mag}$ for the $B$ data and from 0.006 to $0.009 \mathrm{mag}$ for the $V$ data. The check star for X Cyg, HD $196093=47 \mathrm{Cyg}$, was found to vary with an amplitude of 0.04 mag over about 80 days.

The periods that best fit just our APT data for SV Vul and S Vul are 45.036 and 68.500 days, respectively. A recent analysis of the period behaviour of SV Vul (Szabados 1991) has predicted a smaller period for this Cepheid in our observed time interval. S Vul is the faintest Cepheid in our present sample $(V \sim 8.8$ at light maximum) and the scatter in our light curve has not yet permitted a better period determination.

An analysis of a large portion of our APT observations of X Cyg and T Mon (Heiser and Cooper 1991) yielded periods of 16.377 and 27.010 days, respectively. Should these periods be confirmed using all our current APT data, then they would indicate period decreases for both these Cepheids. T Mon is a known binary system and $\mathrm{Cyg}$ has velocity variations which may be interpreted as being due to duplicity. A preliminary analysis of our APT data for Y Oph shows a period of 17.1269 days; in good agreement with the value determined by Szabados (1989).

\section{References:}

Heiser, A.M., Cooper, J.C., 1991, J. Tenn. Acad. Sci. 66, 69

Szabados, L., 1989, Comm. Konkoly Obs., Budapest, No. 94

Szabados, L., 1991, Comm. Konkoly Obs., Budapest, No. 96 